\title{
PRINCIPALES HONGOS FITOPATOGENOS ASOCIADOS A BULBOS ALMACENADOS DE AJO ELEFANTE (Allium ampeloprasum var. holmense) DE LA ZONA DE QUILLOTA Y NOGALES (CHILE)
}

\author{
(Main phytopathogenic fungi associated to stored bulbs of "ajo elefante" (Allium \\ ampeloprasum var. holmense) from the zone of Quillota and Nogales (Chile)) \\ *Ximena A. Besoain, *Rodrigo Véjar,A. \& **Eduardo Piontelli, L. \\ Facultad de Agronomía, Universidad Católica de Valparaíso, \\ Casilla 4-D, Quillota, Chile. \\ **Universidad de Valparaíso, Escuela de Medicina, \\ Cátedra de Micología, Casilla 92 V, Valparaíso, Chile.
}

Palabras clave: Allium ampeloprasum var. holmense, Penicillium hirsutum, P.aurantiogriseum, Fusarium oxysporum, Embellisia allii, moho azul

Key words: Allium ampeloprasum var. holmense, Penicillium hirsutum, P.aurantiogriseum, Fusarium oxysporum, Embellisia allii, blue mould

\section{RESUMEN}

Se presentan los primeros antecedentes fitopatológicos referentes a una prospección de hongos presentes en bulbos almacenados y plantas de Allium ampeloprasum var. holmense (ajo elefante) provenientes de las zonas de Quillota y Nogales (V Región).

A partir de bulbos con diferentes lesiones,se realizaron aislamientos fúngicos correspondientes a los siguientes taxa: Penicillium hirsutum, P.aurantiogriseum, P.echinulatum, P.funiculosum, P.rugulosum, Fusarium oxysporum y Embellisia allii. Estos se inocularon para efectuar pruebas de patogenicidad; tanto en bulbos como en plantas en maceta (salvo Penicillium spp.).

Los resultados obtenidos en bulbos almacenados indicaron que el principal problema en las muestras analizadas fue Penicillium hirsutum, asociado a lesiones inicialmente hendidas, secas, rodeadas de un halo blanquecino, presentando al cabo de 7 días una pudrición blanda acuosa que involucraba la totalidad del bulbo, apareciendo posteriormente el moho azul en las lesiones. Los problemas de importancia secundaria para esta especie vegetal en la zona estudiada fueron Fusarium oxysporum y Embellisia allii.

El principal problema en las plantas en maceta fue E. allii, el cual bajo condiciones de humedad previa y en períodos cercanos a la cosecha, afectó al bulbo cubriéndolo de un micelio negro.
ABSTRACT

The preliminary phytopathogenic data related to the presence of fungi in stored bulbs and plants of Allium ampeloprasum var: holmense (ajo elefante) studied in the zones of Quillota and Nogales (V Región) are issued in this paper.

Considering bulbs having different lesions, fungal isolations were carried out corresponding to the following taxa: Penicillium hirsutum, P.aurantiogriseum, P.echinulatum, P.funiculorum, P.rugulosum, Fusarium oxysporum and Embellisia alli. These were inoculated in order to make pathogenecity tests, both in bulbs and in plants kept in plant-pots (except Penicillium spp.)

It was concluded that the main problem in the samples of stored bulbs was Penicillium hirsutum, associated to initially split and dry lesions surrounded by a whitish circle which developed into a soft watery rot after 7 days, involving the whole bulb and exhibiting further a blue mould on the lesions. Problems having a secondary significance for this vegetal species in the zone under study, were Fusarium oxysporum and Embellista allii.

E.allii was the main problem for plants kept in plant-pots since under conditions of previous humidity and near harvest time attacked the bulb by convering it with black mycelium. 


\section{INTRODUCCION}

El cultivo del ajo (Allium sativum), constituye en nuestro país una actividad productiva tradicional, lo que ha dado origen a numerosas investigaciones, desarrollándose el cultivo desde Arica a Magallanes (Pihán, 1987).Sin embargo, en los últimos años existieron expectativas de un nuevo cultivo como alternativa de consumo interno y como producto de exportación (Torres, 1994) y con aptitudes como producto deshidratado de calidad aceptable para consumo nacional (Figuerola \& Esteve, 1977). Este cultivo conocido como ajo elefante (Allium ampeloprasum var. holmense (Mill.) Aschers \& Graeb), ha sido tradicionalmente cultivado en la zona de Chiloé, $\mathrm{X}$ región, cuyo nombre vulgar es ajo chilote, elefante o blandino y corresponde al grupo denominado vulgarmente ajo de cabeza grande ( Jones \& Mann, 1963). Considerando estos aspectos su cultivo se desplazó más al norte, a las zonas de Quillota y Nogales; extrapolándose las tecnologías obtenidas para el ajo común (Allium sativum), pero sin lograr los resultados deseados. Por tal motivo es importante la generación de tecnologías necesarias tendientes a solucionar los problemas que limitan su cultivo en la zona central.

En el último tiempo se han detectado problemas de pudrición lo que ha motivado a diferentes agricultores a disminuir la superficie plantada con Allium ampeloprasum. En base a estos antecedentes se estableció este estudio, que pretende entregar información preliminar de aislamientos de bulbos y plantas enfermas (Figuras 1-2), identificación de los hongos involucrados y la realización de las correspondientes pruebas de patogenicidad.

\section{MATERIALES Y METODOS}

La investigación se llevó a cabo en el Laboratorio de Fitopatología dependiente de la Facultad de Agronomía de la Universidad Católica de Valparaíso, ubicado en la localidad de La Palma, comuna de Quillota, V Región.

Aislamiento de los hongos. Se realizaron aislamientos a partir de material vegetal correspondiente a la especie vegetal Allium ampeloprasum var. holmense, bajo condiciones de asepsia, en cámara de flujo laminar, utilizando luz ultravioleta como método de desinfección física del área de trabajo. El material vegetal provenía principalmente de las zonas de Quillota y Nogales, el cual fue previamente seleccionado desde bulbos que representaban una superficie aproximada de 10 hectáreas.

Del material vegetal enfermo, se extrajeron trozos de tejido a partir de la zona de avance, los que se desinfectaron con hipoclorito de sodio al 1\% por 30 segun- dos, y luego lavados con agua destilada estéril (ADE), secados y sembrados en placas con los medios de cultivo agar papa dextrosa acidulado (APDA), y agar Czapeck (CZA), e incubadas en oscuridad a $22^{\circ} \mathrm{C}$ en estufa de cultivo. Entre los 7 y 10 días de incubación se obtuvieron colonias correspondientes a los géneros Penicillium, Fusarium y Embellisia. A partir de cultivos puros, se procedió a su identificación y conservación, para poder realizar las pruebas de patogenicidad correspondientes.

\section{Pruebas de patogenicidad en bulbos de postcosecha} Para evaluar la patogenicidad de cepas correspondientes a los géneros Penicillium, Embellisia y Fusarium, se preparó una suspensión de conidios en 10 $\mathrm{ml}$ de ADE en tubos de ensayo. Posteriormente, se midieron las concentraciones de las diferentes muestras a inocular con observaciones al microscopio óptico, empleándose el hematocitómetro de Bauer. La inoculación se realizó en bulbos aparentemente sanos de ajo elefante, los que se sumergieron sin catáfilas en alcohol durante tres minutos, luego en hipoclorito al 1\% por ocho minutos y lavados en abundante ADE. Posteriormente se secaron en papel estéril, desarrollándose todo el proceso de inoculación en cámara de flujo laminar.

Para la prueba de patogenicidad se emplearon 13 cepas correspondiente al género Penicillium (Cuadro 1), 5 cepas de Fusarium oxysporum y 2 cepas de Embellisia allii, empleándose la siguiente metodología: se tomaron tres bulbos sin catáfilas aparentemente sanos de A. ampeloprasum por tratamiento (cada una de las cepas obtenidas corresponde a un tratamiento más el control), los que fueron desinfectados y tratados sin y con heridas (efectuadas con aguja hipodérmica), siendo posteriormente inoculados con 4,5 ml de una suspensión de conidios con una concentración de 1-2 x10-6 ufc/ ml. Luego se colocaron en cámara húmeda, dentro de una estufa de cultivo a $25^{\circ} \mathrm{C}$ en oscuridad. Se realizaron observaciones diarias, tomándose como índice de comparación el desarrollo de las lesiones en el tiempo, con los respectivos testigos, los que fueron sólo inoculados con ADE.

Pruebas de patogenicidad en plantas. Se prepararon contenedores de polietileno negro de un volúmen de dos litros, los que fueron llenados con una mezcla de substrato (suelo, arena,tierra de hoja; 1:1:1) esterilizado con vapor a $80^{\circ} \mathrm{C} / 30^{\prime}$. En ellos se plantaron bulbos de A.ampeloprasum previamente desinfectados, para así obtener plantas aparentemente sanas, las que fueron inoculadas con soluciones preparadas a partir de 5 cepas de Fusarium y 2 cepas de Embellisia. Se excluyó Penicillium por ser un patógeno que afecta al bulbo en postcosecha.

El inóculo se preparó con $150 \mathrm{ml}$ de ADE, el hongo se cultivó en APDA y posteriormente, para 
homogenizarlo se sometió la mezcla a una licuadora por cinco minutos, midiéndose la suspensión de propágulos por medio del hematocitómetro de Bauer, obteniéndose las concentraciones de las diferentes suspensiones. Estas fluctuaron entre $1-2 \times 10^{6}$ propágulos/ ml. Se agregó $50 \mathrm{ml}$ de solución por planta, mientras que a los testigos se les agregó sólo ADE.

Las plantas fueron evaluadas visualmente en forma periódica (dos días por semana), siendo regadas constantemente. Para describir los posibles síntomas presentes en ellas, se compararon con los testigos de las diferentes cepas, y a partir de plantas con síntomas se efectuaron reaislamientos, para ser sometidos a identificación y demostrar si correspondían a las cepas inoculadas, mediante las metodologías de aislamiento e identificación descritas a continuación.

\section{Metodología de identificación}

Para los aislamientos e identificación de las cepas del género Penicillium, se utilizó la metodología y claves propuestas por Pitt $(1979,2000)$, que consisten en sembrar el hongo en diferentes medios de cultivo (AEM, CYA, G25N), para determinar el diámetro de las colonias, color, exudados, temperaturas máxima - mínima de crecimiento y morfología, manteniendo los cultivos a $25^{\circ} \mathrm{C}$ y evaluándose a los siete días.

Para el género Fusarium se sembraron muestras en los medios PDA para observar la coloración de las colonias y el agar clavel ( Nelson et al., 1983) para determinar las características micromorfológicas del hongo. Para el género Embellisia, se estudiaron las características macro y microscópicas en cultivos comparandolas con la monografía de Ellis (1976).

\section{RESULTADOS Y DISCUSION}

1.- Aislamientos fúngicos e identificación. A partir de lesiones secas hendidas, presentes en bulbos de ajo elefante (Figura 1, 2), se aislaron entre los 7 y 10 días de incubación 18 cepas de Penicillium, 14 de $\boldsymbol{P}$. hirsutum (descritas en punto 2), 1 de $\boldsymbol{P}$. aurantiogriseum, 1 de $\boldsymbol{P}$. echinulatum 1 de $\boldsymbol{P}$. funiculosum, 1 de $\boldsymbol{P}$. rugulosum.

Desde la base de los bulbos y a partir de lesiones de color café claro, se aislaron 5 cepas de Fusarium, todas correspondientes a F.oxysporum. A partir de catáfilas que presentaban un claro daño cosmético producido por un moho negro que las recubría, se aislaron 2 cepas de Embellisia allii (Descritas en punto 2).

\section{2.- Descripción de las principales cepas \\ Penicillium hirsutum Dierckx (Figura 4) \\ $=$ Penicillium corymbiferum Westling (Para otros si- nónimos ver Pitt, 2000).}

Colonias de crecimiento rápido en agar CYA y MEA, en CYA entre 28-32mm de diámetro en 7 días y en MEA 31a $34 \mathrm{~mm}$. El aspecto en MEA, fue variable, pero generalmente de textura aterciopelada y planas, a veces surcadas o de aspecto granular fasciculado a coremiforme; en CYA color variable desde verde amarillento a amarillo brillante, con márgenes blancos. Exudado presente de color café violáceo y pigmento soluble color café naranja, reverso café naranja oscuro a rojo café. Conidióforos rugosos, terverticilados, asimétricos, largos 220-460 $\mu \mathrm{m}$ que nacen generalmente en fascículos, con ramas típicamente rugosas al igual que el racimo terminal de métulas, 8-13 x 2-3 $\mu \mathrm{m}$,. Fiálides ampuliformes acerosas, lisas, 7,2 a 9,5 × $2 \mu \mathrm{m}$, conidios principalmente esféricos a veces elipsoides, lisos a finamente rugosos que nacen en cadenas desordenadas 3,5 - $4 \mu \mathrm{m}$. Ninguna cepa creció a a $37^{\circ} \mathrm{C}$.

Los aislamientos analizados, presentaron variaciones en color, exudado y producción de coremios en cultivo. Esta especie presenta semejanzas morfológicas con: P.allii, P.hordei, P.albocoremium, P.viridicatum y P.aurantiogriseum (Pitt, 2000, Samson et al., 2000). Es una especie que generalmente se encuentra asociada a bulbos de Lilaceae.

Embellisia allii (Campanile) Simmons (Figura 6). = Helminthosporium allii Campanile, 1924

Colonias en agar papa dextrosa de crecimiento rápido, de color café a negro, de aspecto pulverulento a aterciopelado, profusamente esporuladas. Micelio dematiáceo septado, liso con conidióforos derechos o sinuosos en el ápice, septados, lisos, de paredes gruesas,40-80 x 5-8 $\mu \mathrm{m}$. Conidios elipsoidales o subcilíndricos, no en cadenas, lisos, de color café oscuro cuando maduros, generalmente con 3 a 6 septos transversales oscuros, a veces con algún septo longitudinal u oblicuo, 21-30 x 10-13 $\mu \mathrm{m}$. Los conidios dan origen también a conidióforos secundarios cortos, que originan nuevos conidios.

Los aislamientos presentaron las características de la especie. Es poco común en el ambiente, salvo en bulbos de Liliaceae y los primeros registros en Chile parecen corresponder a los efectuados por Sanz (1978), en la misma zona (Nogales) donde al parecer el hongo permanece como endémico.

El cultivo de Penicillium aurantiogriseum Dierckx, presentó características bien definidas que permitieron su inclusión en la especie, sin embargo, presenta semejanzas con $\boldsymbol{P}$. cyclopium, P.viridicatum y P.aurantiocandidum (Samson et al.,2000). Es una especie de amplia distribución en cereales y otros hábitat y muy común en nuestro ambiente.

Los cultivos de Penicillium echinulatum Fassat., Penicillium rugulosum Thom y Penicillium funiculosum 
Thom, presentaron las características descritas en la literatura, las 2 primeras especies son poco comunes en el ambiente, mientras la última es cosmopolita, presente en el suelo y cereales y también de amplia distribución en nuestro ambiente.

Las 5 cepas deFusarium oxysporum Schlecht.emend. Snyd.\& Hans., fueron estables en cultivo y no presentaron mayores variaciones (Nelson et al.,1983). Esta especie es de amplia distribución como saprófito del suelo, pero al mismo tiempo ciertas cepas pueden comportarse como fitopatógenas. Común en cereales, frutas, bulbos o causando pudrición en almacenamiento.

3.- Pruebas de patogenicidad en postcosecha. De acuerdo con los resultados obtenidos en las pruebas de patogenicidad con las cepas del género Penicillium (Cuadro1), sólo se obtuvo daño en el caso de los tratamientos con heridas. Esto confirma que estas especies no son capaces de penetrar los tejidos sin la presencia de éstas. En los tratamiento con heridas, se presentaron diferentes niveles de virulencia, siendo la especie más agresiva $\boldsymbol{P}$. hirsutum Dierckx, con desarrollo de lesiones a los cuatro días posteriores a la inoculación, posiblemente por su mayor resistencia a la allicina, compuesto antibiótico producido por las especies del género Allium, como respuesta a las lesiones (Salley \& Hansen,1962).

Los síntomas inicialmente consistieron en una lesión hendida, seca, rodeada de un halo blanquecino, derivando al cabo de siete días en una pudrición blanda acuosa que cubría prácticamente todo el bulbo. Dentro de las diferentes cepas de $\boldsymbol{P}$. hirsutum no se apreciaron claras diferencias de virulencia, salvo en la cepa PH2 (Figura

Cuadro. 1. Patogenicidad (con heridas) de diferentes especies y cepas del género Penicillium en bulbos de Allium ampeloprasum.

\begin{tabular}{|c|c|c|}
\hline Especies & Cepas & Patogenicidad* \\
\hline P. hirsutum & 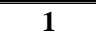 & ++ \\
\hline P. hirsutum & 2 & +++ \\
\hline P. hirsutum & 3 & ++ \\
\hline P. hirsutum & 4 & ++ \\
\hline P. hirsutum & 5 & ++ \\
\hline P. hirsutum & 6 & ++ \\
\hline P. hirsutum & 7 & + \\
\hline P. hirsutum & 10 & ++ \\
\hline P. hirsutum & 14 & + \\
\hline P. echinulatum & - & - \\
\hline$P$ aurantiogriseum & - & + \\
\hline P. funiculosum & - & $+/-$ \\
\hline P. rugulosum & - & $+/-$ \\
\hline Testigo(agua estéril) & - & - \\
\hline
\end{tabular}

+ = Levemente virulenta; $++=$ Moderadamente virulenta; +++=Altamente virulento; +/- = Se desarrolla, sin virulencia; - =Sin daño aparente. * 7 días de incubación.
3), donde se apreció una mayor diferencia con respecto a las otras cepas.

Otra de las especies patogénicas aisladas fue $\boldsymbol{P}$. aurantiogriseum Dierck, pero de moderada virulencia con respecto a $\boldsymbol{P}$. hirsutum. Las otras especies inoculadas fueron $\boldsymbol{P}$. funiculosum Thom, P.echinulatum Fassat., P. rugulosum Tom, quienes mostraron sólo un leve desarrollo de micelio y conidios en relación a las otras especies, ya que el daño se restringió sólo a las heridas (Cuadro 1). La mayor virulencia de $\boldsymbol{P}$. hirsutum indica que esta especie es el principal agente causal de esta patología. A nivel mundial, P.hirsutum y P.aurantiogriseum complex, han sido descritos como causantes de la enfermedad en ajo (Scwartz y Mohan,1995). Bruna (1985), en Chile determina a $\boldsymbol{P}$. corymbiferum (ahora denominado $\boldsymbol{P}$. hirsutum) como causante del moho azul en el caso de Allium sativum. Por otro lado, Illanes y Acuña (1993), en sus experiencias en la zona sur del país también asocian a $\boldsymbol{P}$. hirsutum como causante, tanto en $A$. sativum como en A. ampeloprasum, sin probar la patogenicidad de las cepas aisladas. Por lo tanto, para $P$. aurantiogriseum, sería la primera determinación a nivel nacional asociada al género Allium. Dentro de las especies causantes de esta enfermedad Schwartz y Mohan (1995), incluyen a $\boldsymbol{P}$. funiculosum (en A. sativum). Sin embargo, nuestros datos difieren en su capacidad patogénica para A. ampeloprasum. Cabe señalar que sólo se logró un aislamiento de esta especie asociada a daños en postcosecha.

Embellisia allii, mostró una virulencia leve en el tratamiento con heridas (Figura 5), restringiéndose el crecimiento a las lesiones, lo que podría deberse a algún tipo de inhibición por sustancias producidas por $A$. ampeloprasum en respuesta a las heridas, ya que para el tratamiento sin heridas se logró un mayor desarrollo del moho, cubriendo una zona más amplia del bulbo, a diferencia de los trabajos realizados por Sanz (1978) en ajo rosado (Allium sativum), donde las heridas favorecieron el desarrollo del hongo. De las dos cepas inoculadas la número uno mostró un mayor desarrollo (moderadamente virulento), cubriendo una mayor superficie en los bulbos tratados, mientras la 2 fue menos virulenta.

Los tratamientos realizados con las 5 cepas de la especie Fusarium oxysporum se caracterizaron por el desarrollo de síntomas consistentes en una pudrición blanda semiacuosa de los tejidos del bulbo, seguidos por la formación de un micelio blanco algodonoso compacto el cual creció desde la base del bulbo hacia el ápice del mismo. Todas las cepas mostraron una virulencia moderada a los seis días, no existiendo grandes diferencias entre éstas con respecto al grado de virulencia mostrado en ambos tratamientos, sin embargo, el tratamiento con heridas fue más favorable para el desarrollo del hongo, a diferencia del tratamiento sin heridas en que el hongo se 

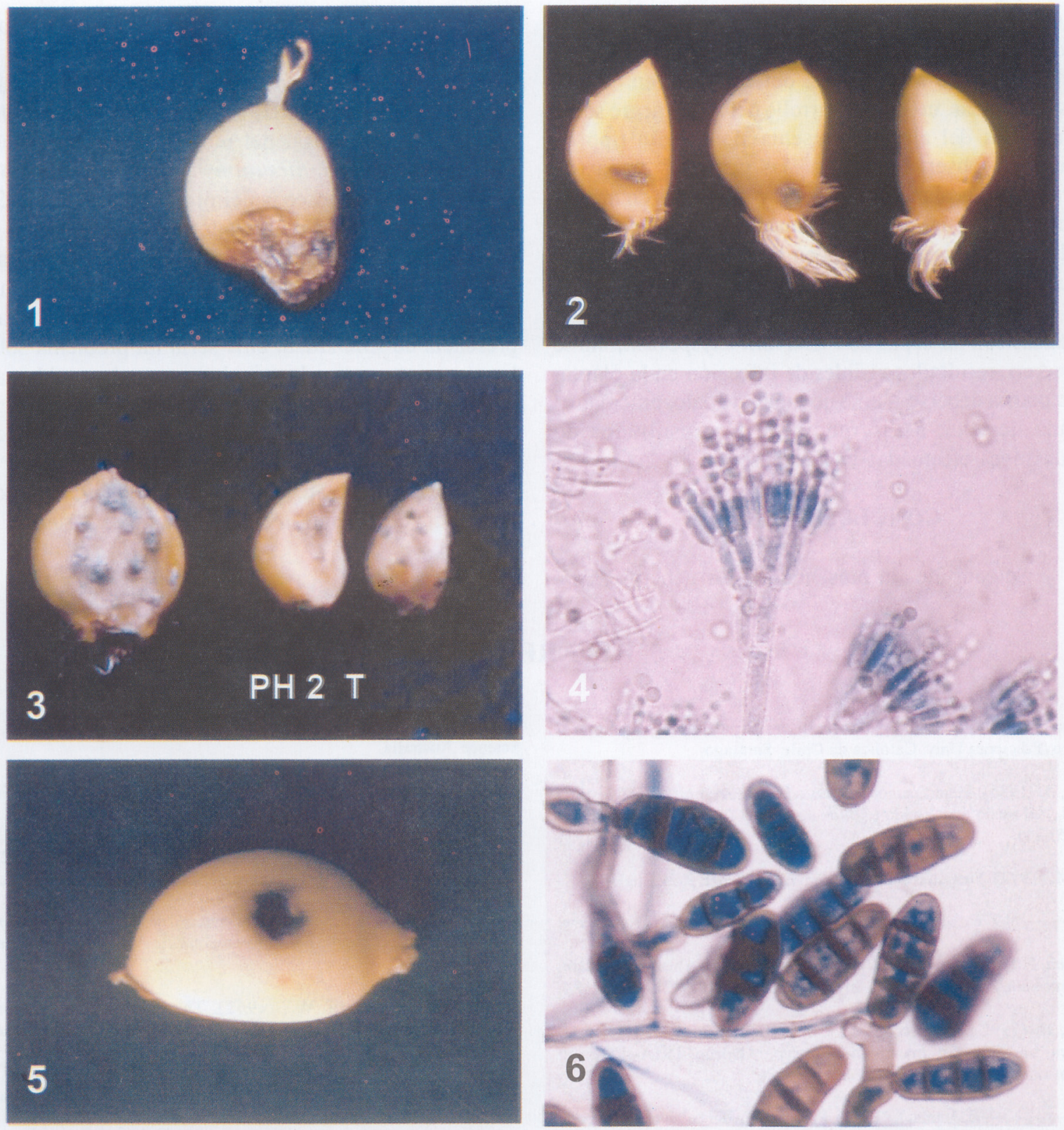

Figura 1- 1.- . 1, 2.- Bulbos de ajo elefante naturalmente infectados con Penicillium hirsutum, presencia de lesiones hendidas y secas. 3.- Bulbos de ajos elefante inoculados mediante herida, con cepa PH2 de $\boldsymbol{P}$. hirsutum, donde se aprecian lesiones blandas y acuosas (7 días post inoculación). 4.- P. hirsutum, conidióforo y conidios, 1000x. 5.- Lesión desarrollada por Embellisia allii en bulbo de ajo elefante inoculado mediante herida. 6.- E. allii, conidióforos y conidios, 1000x.

desarrolló sólo en la base del bulbo, siendo clasificado como un daño leve.

4.- Patogenicidad en plantas en maceta. En las pruebas de patogenicidad efectuadas con ajo elefante desarrollado en macetas, sólo se obtuvo plantas afectadas con el tratamiento con inóculo de Embellisia allii, las cuales mostraron los signos característicos de la enfermedad, con presencia del moho negro en las catáfilas exteriores al final del período de cultivo, desmejorando la calidad visual del producto, aunque ésto sólo se produjo en plantas sometidas a riegos tardíos. Estas son las características típicas de la enfermedad que afecta a Allium sativum descritas por Bruna (1991), denominada cabeza negra o helmintosporiosis (Apablaza, 2000). Además, se demues-tra que estos bulbos son más susceptibles por presentar túnicas blancas, como lo afirman Jones \& Mann (1963), Bruna (1991) y Smith (1992). También se confirmó lo observado por Bruna (1991) y Messiaen et al., (1995), en lo referente a que el 
hongo se desarrolla con humedad y temperaturas altas en el suelo. En la experiencia reali-zada, las plantas fueron sometidas a estas condiciones, obteniéndose en el caso de las inoculadas, un gran desarrollo del moho negro sobre las cubiertas del ajo (no presentando ningún tipo de pudrición), observándose en las primeras etapas de desarrollo de las plantas inoculadas y cultivadas en maceta los síntomas característicos de amarillamiento y posterior secado de las hojas, descritos en literatura. En el testigo no hubo desarrollo del hongo.

En el caso de las pruebas de patogenicidad efectuadas con las cepas de Fusarium oxysporum, no se obtuvieron resultados visibles de infección en la planta, lo que podría deberse a un bajo grado de patogenicidad o pérdida de ésta, a diferencia de los resultados de inoculaciones efectuadas en postcosecha.

\section{CONCLUSIONES}

Se constató la presencia de los géneros Peni- cillium y Fusarium como causantes de problemas de postcosecha en bulbos de Allium ampeloprasum, y de Embellisia allii como problema asociado a plantas cultivadas. Los problemas causados por el género Penicillium, agente causal del moho azul, son atribuibles principalmente a $\boldsymbol{P}$. hirsutum y esto repercute no sólo en la vida de postcosecha del producto, sino también en la calidad del material utilizado como semilla.

Se constató que la fusariosis es provocada por Fusarium oxysporum, la que se presentó como el segundo problema en importancia en postcosecha. La presencia de Embellisia allii sería un problema sólo en las etapas cercanas a la cosecha, causando problemas de presentación del producto.

De acuerdo a éstos resultados, los problemas fitopatológicos que afectan a A. ampeloprasum son similares a los que afectan a Allium sativum, variando la incidencia de las enfermedades en ambos casos.

\section{REFERENCIAS}

Apablaza,G. (2000). Patología de cultivos , epidemiología y control holístico. Ediciones Univ. Católica de Chile, Santiago.

Bruna, A. (1985). Identificación de Penicillium corymbiferum Westling causante del moho azul en ajo (Allium sativum L.). Agricultura Técnica 45(4):353-356.

Ellis, M. (1971). Dematiaceous Hyphomycetes. CMI, Kew.England

(1976). More Dematiaceous Hyphomycetes.CMI,Kew. Kew.

Figueroa, F. \& Estévez, A.(1977). Deshidratación del ajo blandino (Allium ampeloprasum ) del sur de Chile. Investigación Agrícola (Chile) 3: 81-84.

Illanes, B. (1992). Prospección del cultivo del ajo en la X región : I. Especies, tiposde ajo y manejo del cultivo. Osorno, INIA- Estación Experimental Remehue. Boletín Técnico Nº 186.

\& Acuña, I. (1993). Prospección del cultivo del ajo en la X región : II. Problemas fitopatológicos. Osorno, INIA-Estación Experimental Remehue, Boletín Técnico № 195.

Jones, H.A. \& Mann, L.K. (1963). Onions and their allies; botany, cultivation and utilization. New York. Interscience Publishers

Messiaen, D.; Blancard, F.; Rouxel,A. \& Lafon,A. (1995). Enfermedades de las Hortalizas. Madrid. España, Mundi-Prensa.

Mordue, J. (1976). Sclerotium cepivorum. Description of Patogenic Fungi and Bacteria. Commonwealth Agricultural Bureaux. N ${ }^{\circ} 512$.

Nelson, P.E.; Toussoun, T.A. \& Marasas, W.F.O. (1983). Fusarium species. An illustrated manual for identification. The Pennsylvania State Univ. Press. University Park and London.

Pihán, R. (1987). Producción de ajos. IPA Carillanca 6 (1):2-5.

Pitt, J. (1979). The Genus Penicillium and its teleomorphic states Eupenicillium and Talaromyces. London, Academic Press.
Pitt, J. (2000). A Laboratory guide to common Penicillium species. Food Science Australia.

Raper, K. \& Thom, C. (1949). Manual of the Penicillia. Baltimore, Williams Wilkins.

Samson,R.A.; Hoekstra, E.L.; Fisvald, J.C. \& Filtenborg, O.(2000). Introduction to food and airborne fungi (Sixth Edition). CBS, Utrecht.

Sanz, H. (1978).Identificación y patogenicidad de Helminthosporium allii (Campanile) en ajo rosado (Allium sativum L.). Agricultura Técnica 38 : $122-123$

Sayre, R \& Mountain, W. (1962). The bulb and Stem nematode (Ditylenchus dipsaci) on onion in sothwestern Ontario. Phytopathology 52: $510-516$

Schwartz, H. \& Mohan, S. (1995). Compendium of Onion Garlic Diseases. St. Paul, Minnesotta. APS Press.

Smalley, E. \& Hansen, H. (1962). Penicillium Decay of Garlic. Phytopathology 52 : 666-678

Smith, I.; Dunez, J.; Phillips, D.; Lelliot, R.; Archer, S. (1992). Manual de enfermedades de las plantas. Madrid, Mundi-Prensa.

Somerville, P \& Hall, D. (1987). Factors Affecting Sclerotial Germination of Sclerotium cepivorum, Secondary Sclerotia Formation, and Germination Stimulants to Reduce Inoculum Density. Plant Disease 71:229233

Taylor, A. (1971). Introducción a la Nematología Vegetal Aplicada. Italia, FAO.

Torres, S. (1994). Seguimiento fenológico y Agronómico del cultivo del ajo elefante (Allium ampeloprasum) con destino a exportación en predios ubicados en las comunas de Limache y Quillota. Tesis Ing. Agr. Quillota, Universidad Católica de Valparaíso. Facultad de Agronomía. 